\title{
A huge and asymptomatic aortic aneurysm mimicking intra-cardiac tumor
}

Nan-Chun $\mathrm{Wu}^{1}$, Jhih-Yuan Shih ${ }^{1}$, Bor-Chih Cheng ${ }^{1}$, Zhih-Cherng Chen ${ }^{1}$, and Wei-Ting Chang $^{1}$

\author{
${ }^{1}$ Chi Mei Medical Center
}

May 15, 2020

\begin{abstract}
Despite sinus valsalva aneurysm of a well-known pathology, its presence as a cardiac tumor with the RA compression is rare. A 64-year-old man was accidentally identified with a cardiac tumor during the survey of atrial fibrillation. The initial echocardiography and chest computed tomography (CT) displayed a huge mass at right atrium. Surprisingly, the operative findings diagnosed a sinus valsava aneurysm. This case highlights the diagnostic challenges of a huge sinus valsava aneurysm which mimics an intra-cardiac tumor.
\end{abstract}

\section{Hosted file}

Aortic aneurysm mimicking intra-cardiac tumor.doc available at https://authorea.com/users/ 313657/articles/451319-a-huge-and-asymptomatic-aortic-aneurysm-mimicking-intra-cardiactumor 

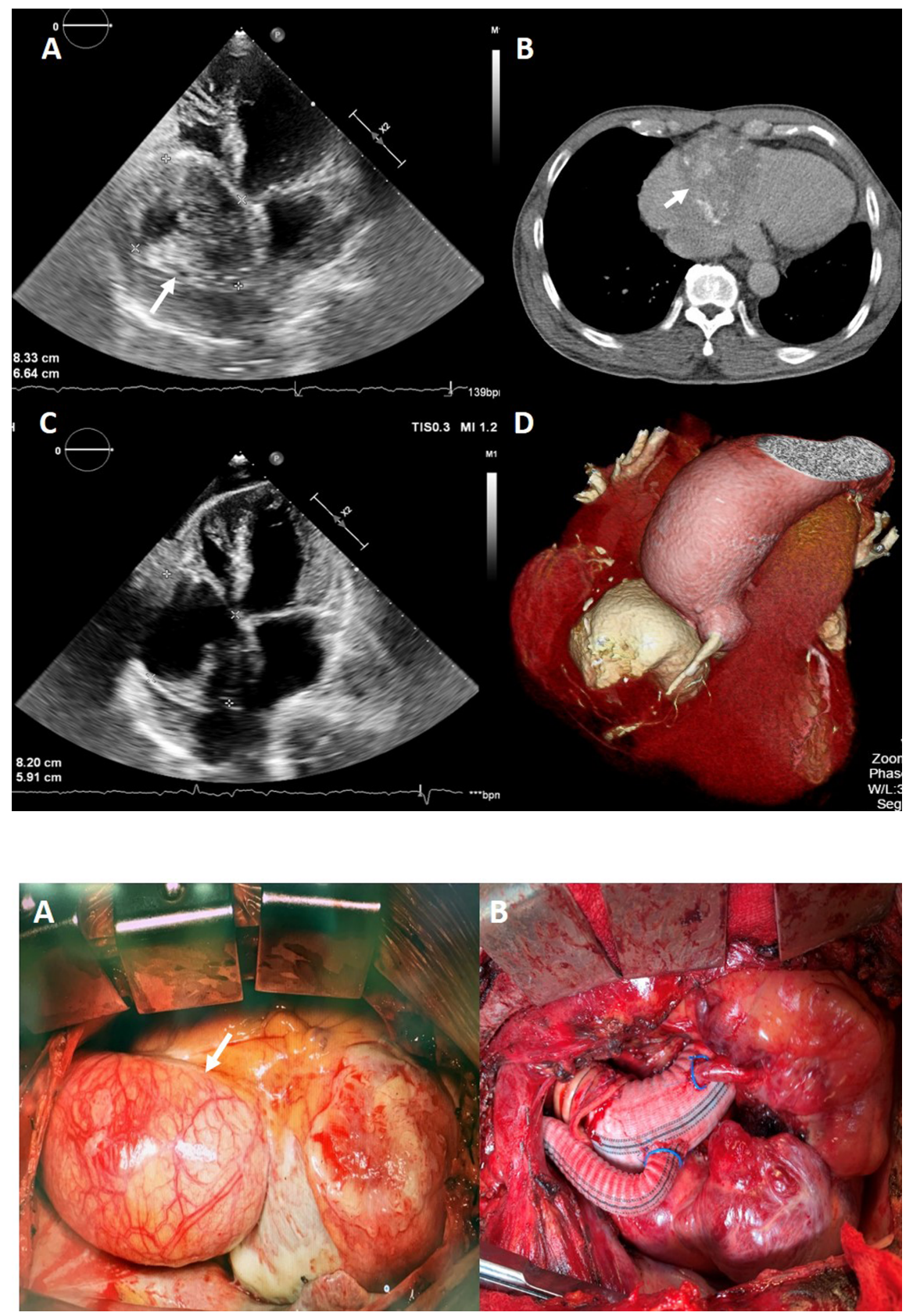


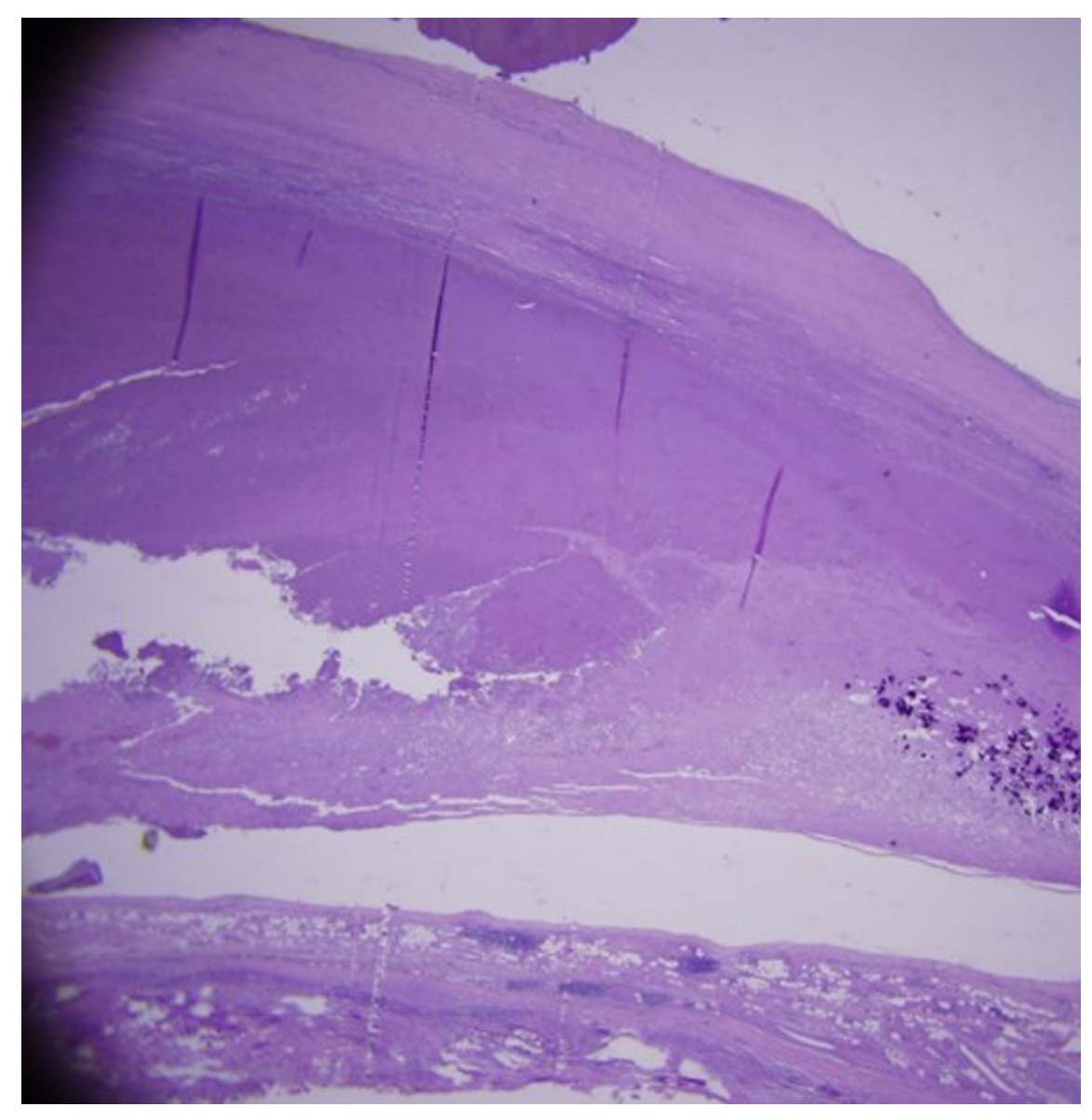

